\section{Asthmakontrolle mit nur einer Inhalation}

\author{
Ciclesonid ist ein neues inhalatives Glukokortikoid zur Behandlung \\ des Asthma bronchiale. Die Substanz wird erst in den Atemwegen \\ in die pharmakologisch aktive Form überführt, was systemische und \\ orale Nebenwirkungen reduziert. In einer Parallelgruppenstudie \\ wurden zwei Dosisstärken von Ciclesonid untersucht.
}

$K_{p}^{2}$ anadische Studienärzte bezogen 329

Patienten mit persistierendem Asth$\mathrm{ma}$ in eine doppelblinde plazebokontrollierte Untersuchung ein. Die Probanden wurden zunächst mindestens 4 Wochen stabil auf ein Dosisäquivalent von 400 bis $800 \mu \mathrm{g} / \mathrm{d}$ Beclometason eingestellt. Dann erhielten sie über 12 Wochen entweder $160 \mu \mathrm{g} / \mathrm{d}$ Ciclesonid $(\mathrm{n}=112)$, $640 \mu \mathrm{g} / \mathrm{d}$ Ciclesonid $(\mathrm{n}=112)$ oder Plazebo $(n=110)$, jeweils einmal morgens.
Unter beiden Ciclesonid-Dosierungen blieb der morgendliche Peak Expiratory Flow (PEF, maximale Atemstromstärke) stabil, während er unter Plazebo signifikant absank. Ein ähnliches Bild ergab sich bei der Einsekundenkapazität $\left(\mathrm{FEV}_{1}\right)$ und der forcierten Vitalkapazität. Patienten unter Plazebo schieden auch signifikant häufiger wegen Unwirksamkeit der Medikation aus (63\%) verglichen mit Patienten unter $160 \mu \mathrm{g} / \mathrm{d}$ Cic- lesonid (30\%) oder unter $640 \mu \mathrm{g} / \mathrm{d}$ Ciclesonid (31\%). Signifikante Unterschiede in Wirksamkeit und Sicherheit zwischen den beiden Verumdosierungen waren nicht zu erkennen. Die Serumund 24-h-Urinkonzentrationen von Cortisol blieben konstant, die Verträglichkeit war gut, eine Candidiasis trat in keinem Fall auf.

Fazit: Bei Patienten mit mildem bis moderatem persistierenden Asthma ist inhalatives Ciclesonid bereits in der Dosis von einmal täglich morgens $160 \mu \mathrm{g}$ wirksam und sicher. Das Nebenwirkungsprofil entspricht dem von Plazebo. $\quad b k$

Chapman KR et al. Maintenance of asthma control by once-daily inhaled ciclesonide in adults with persistent asthma. Allergy 2005; 60: 330-7

\title{
Wie lange wirken Glukokortikoide?
}

Inhalative Glukokortikoide sind aufgrund ihrer entzündungshemmenden Wirkung Mittel der Wahl in der Anfallsprophylaxe des allergischen Asthmas. Doch zu der Frage, wie lange diese protektive Wirkung anhält, gibt es bislang nur wenige Untersuchungen.

드. in Forscherteam ließ 26 Patienten mit leichtem allergischen Asthma über eine Woche hinweg $200 \mu \mathrm{g}$ Budesonid, $200 \mu \mathrm{g}$ Fluticason oder Plazebo jeweils zwei Mal pro Tag inhalieren. 12 Stunden nach Absetzen der Therapie unterzogen sich die Teilnehmer inhalativen Provokationstests mit dem Allergen, gegen das sie im Hauttest am stärksten reagiert hatten. Nach einer Pause von 3 Wochen wiederholten sich Therapieschema und Untersuchungen im Cross-overDesign mit einer anderen Medikation.

Im Vergleich zu Plazebo ergab sich für die Patienten, die Fluticason inhaliert hatten, eine signifikant geringere Reduktion des $\mathrm{FEV}_{1}$ unmittelbar nach Allergenprovokation. Dies war für Budesonid nicht nachweisbar. Der maximale Abfall des $\mathrm{FEV}_{1}$ bei der Frühreaktion betrug nach Fluticasongabe im Mittel 19\%, nach Budesonidgabe 23\% und nach Plazebo $26 \%$. Berechnete man allerdings die

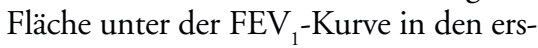

ten 2 Stunden nach der Provokation, waren keine Unterschiede mehr zwischen den beiden Substanzen vorhanden.

Die allergeninduzierte Spätreaktion, ermittelt als Abfall des $\mathrm{FEV}_{1}$-Werts

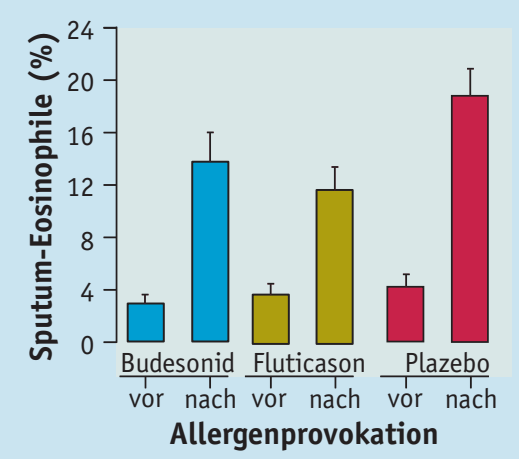

Bei einer Allergenprovokation nach Absetzen einer inhalativen Therapie mit Budenosid, Fluticason oder Plazebo ist die Erhöhung der Eosinophilen im Sputum bei den mit den Glukokortikoiden vorbehandelten Patienten signifikant geringer $(p=0,001)$.
3 bis 7 Stunden nach Allergenexposition, fiel bei den mit Glukokortikoiden behandelten Probanden im Vergleich zu Plazebo signifikant geringer aus. Dies galt allerdings nur für die Patienten, bei denen es in der Frühreaktion zu einer mehr als 15\%igen Absenkung des FEV gekommen war.

Beim Methacholintest zur Untersuchung der allergenvermittelten bronchialen Hyperreagibilität 24 Stunden nach Allergenexposition ergaben sich keine signifikanten Unterschiede zwischen den Substanzen. Allein beim Anstieg der Eosinophilen im Sputum nach Allergenprovokation konnte eine Langzeitwirkung der Therapie mit den inhalativen Glukokortikoiden gezeigt werden (Abb.).

Fazit: Die inhalativen Glukokortikoide Budesonid und Fluticason zeigten in dieser Studie 12 Stunden nach Absetzen nur noch teilweise protektive Wirkung gegen allergisches Asthma. Die Autoren sehen darin die Bestätigung für die Notwendigkeit einer zweimal täglichen Gabe dieser Substanzen.

Subbarao $\mathrm{P}$ et al. Protection by budenoside and fluticasone on allergen-induced airway responses after discontinuation of therapy. J Allergy Clin Immunol 2005; 115: 745-50 